\title{
Comparison of Pain Response to Venepuncture Versus Heel Lance Blood Sampling in Term Neonates
}

\author{
Shrestha M' ${ }^{1}$ Adhikari RK² \\ ${ }^{1}$ Dr. Manish Shrestha, MD, ${ }^{2}$ Dr. Ramesh Kant Adhikari, MD, Department of Paediatrics, Institute of Medicine, Tribhuvan \\ Univerity, Maharajgunj, Kathmandu, Nepal
}

Address for correspondence : Dr. Manish Shrestha, E-mail: manishrestha@msn.com

\begin{abstract}
Introduction: Pain in neonates is largely underestimated and neglected. Pain experience can alter clinical outcome, brain development and subsequent behavior in newborns. Numerous newborns undergo blood sampling routinely in nurseries/NICUs and these procedures are often done without pain relieving measures. Heel lancing and venepuncture are two common procedures for blood sampling in neonates. The objective of this study was to compare pain response to venepuncture versus heel lance in full term neonates. Materials and Methods: A comparative observational study was conducted among 200 term neonates who were undergoing blood sampling for bilirubin or glucose estimation. Neonates were randomly assigned to heel lance (HL) and venepuncture (VP) groups with 100 babies in each group. During the procedure, pain was assessed by Neonatal/Infant pain Scale (NIPS). Heart rate (HR) and oxygen saturation $\left(\mathrm{SpO}_{2}\right)$ were continuously monitored 5 minutes prior to procedure and upto 5 minutes after the procedure. Results: The median NIPS score in HL and VP were 7 and 3.5 respectively which showed statistically significant ( $p=0.0001)$ higher level of pain experience in HL than in VP. During the procedure, both the groups ( $\mathrm{HL}$ and VP) showed significant changes in heart rate and oxygen saturation i.e., increase in $\mathrm{HR}(p=0.0001)$ and decrease in $\mathrm{SpO}_{2}(p=0.0001)$, however the increase in heart rate and decrease in oxygen saturation were significantly more in $\mathrm{HL}$ than in VP $(p=0.0001)$. Conclusion: Neonates perceive pain as demonstrated by Neonatal Pain Scale and venepuncture is the less painful procedure than heel lancing for blood sampling in neonates.
\end{abstract}

Key words: Heel lance, Neonates, NIPS, Pain, Venepuncture

\section{Introduction}

P ain is an unpleasant sensory and emotional experience associated with actual or potential tissue damage or described in terms of such damage. Though pain is a subjective phenomenon but inability to communicate it does not negate the possibility that an individual is experiencing pain and is in need of appropriate pain relieving treatment ${ }^{1}$. Numerous newborns routinely undergo blood-letting procedures in nurseries/NICU for diagnostic and therapeutic purposes but usually these procedures are done without pain preventing or relieving measures. The misconception that the infant does not perceive pain due to various reasons (incomplete myelination,

Manuscript received: $7^{\text {th }}$ January 2012.

Reviewed: $17^{\text {th }}$ March 2012

Author Corrected: $16^{\text {th }}$ April 2012

Accepted for Publication: $30^{\text {th }}$ April 2012 immature central nervous system, etc.) has been dispelled and it has been emphasized that even extremely preterm babies also perceive pain and suffer from more serious consequences than older children and adults ${ }^{2-4}$. Unrelieved pain in neonates may result in increased morbidity and length of stay in nurseries/ $\mathrm{NICU}$, altered immune function, exaggerated pain in later life and altered psychosocial development ${ }^{4-6}$.

As neonates cannot self-report pain, assessment of pain in neonates has to be relied on behavioral and physiologic reactions to pain. Physiologic markers usually include changes in heart rate, respiratory rate, blood pressure, and oxygen saturation ${ }^{7,8}$. Behavioral reactions include changes in facial expressions, body movements, and crying; however these may be absent in some early preterm, neurologically impaired, pharmacologically treated, or intubated neonates ${ }^{7,9,10}$. The objective pain assessment require 
a multidimensional tool that encompasses these parameters. Various pain assessment scales have been developed and validated for this purpose. These are based on either behavioral changes or physiological variations or a combination of both. The latter provides a better estimate of infant pain ${ }^{3}$.

Heel lancing and venepuncture are the common procedures for blood sampling. The former is the conventional method in neonates for measurement of bilirubin, glucose and screening for metabolic disorders ${ }^{11}$. Heel lancing is painful and various pharmacological and non-pharmacological measures (like breastfeeding, non-nutritive sucking, sucrose etc.) are being investigated for pain management however no effective and practical methods have been developed till date to alleviate pain from heel lancing ${ }^{11,12}$. The procedure of heel lancing raises concerns apart from pain, which include chances of collecting hemolyzed sample, inadequate sample from single prick and chances of puncturing calcaneus bone leading to osteochondritis ${ }^{11}$. Venepuncture, on the other hand, a common procedure in older children and adults, has a reduced risk of a hemolyzed or clotted sample, increased sample volume and possibly less pain. However a skilled phlebotomist is needed to perform the procedure ${ }^{11,12}$. Usually no efforts are made to assess or mitigate pain during these procedures. This study is an attempt in that direction.

The objective of this study was to compare the pain response to venepuncture versus heel lance in full term neonates.

\section{Materials and Methods}

A randomized comparative observational study was carried out over a period of six months from January to June 2010 in maternity ward at Tribhuvan University Teaching Hospital (TUTH), Kathmandu. 200 term newborns (age more than 12 hours and less than 8 days) with an APGAR score of 7 or more at 5 minutes of birth and who were undergoing blood sampling for bilirubin or glucose estimation were randomly assigned to heel lance $(\mathrm{HL})$ and venepuncture (VP) groups each having 100 babies. Babies with significant morbidity like birth asphyxia, major congenital malformations or any illness were excluded. Babies who received multiple pricks $(>1)$ during blood sampling and whose mother had a history of receiving epidural analgesia for delivery or any form of opioids during pregnancy or labor and whose parents refused consent were also excluded.

An informed consent was taken from the parents prior to the procedure. Randomization was done by lottery method. The parents were asked to draw a chit containing allocation group from the box. All neonates were fed 1-2 hours before procedure and they were in a state of sleeping lightly or awake and resting quietly. The blood drawing procedure was carried out by the nurses on duty. Before the procedure, the test area was warmed for 1 minute by gently placing it between the warm hands of the nurse, thereafter the skin was cleansed with disinfectant (spirit swab) and awaited for two seconds. After penetrating the skin with either a venepuncture needle or a heel lance, the infants were left undisturbed for 30 seconds during which time no effort was made to induce blood flow. Heel lancing and venepucture were performed by standard microlance and standard 24 gauze needle respectively. The microlance had a very sharp end protruding approximately $3 \mathrm{~mm}$ through lancing device for standard skin depth. Neonatal response to pain was rated on NIPS (Neonatal/Infant Pain Scale). NIPS assess five behavioral parameters (facial expression, cry, motor activities of arms and legs, and state of arousal) and 1 physiological parameter (breathing pattern). For each parameter, score ranges from 0 to 1 except for cry where ranges from 0 to 2. Maximum score is 7 and score more than 3 indicates pain ${ }^{18}$.

Two observers independently rated pain on NIPS. Both the observers were resident doctors with similar professional background; observer 1 being the investigator himself and observer 2 being the other resident doctor unrelated to purpose and outcome of the study. The observer 2 was familiar with each parameter of NIPS. Heart rate and oxygen saturation were continuously monitored for 5 minutes before the procedure to 5 minutes after the procedure by standard pulse oximetry.

\section{Statistical analysis}

Data were analyzed using SPSS statistical software (version 16). Descriptive statistics (mean, median and proportion) were obtained and comparative analysis was done by T-test or chi-square test as appropriate. The NIPS score rated by both the observers were computed separately and interobserver reliability was obtained by using Pearson correlation. Correlations between NIPS and physiological markers of pain were examined by using Spearman rank-order correlations. A $p$-value $<0.05$ was considered significant.

\section{Results}

Table 1 summarizes the demographic characteristics of the neonates studied. There were no significant differences between the groups with respect to any basic data. 
Table 1: Demographic characteristics of study subjects

\begin{tabular}{|l|r|r|r|}
\hline & \multicolumn{1}{|c|}{$\begin{array}{c}\text { HL } \\
(\mathbf{n = 1 0 0 )}\end{array}$} & $\begin{array}{c}\text { VP } \\
(\mathbf{n}=\mathbf{1 0 0})\end{array}$ & $\boldsymbol{p}$-value \\
\hline $\begin{array}{l}\text { Mean age at } \\
\text { intervention (hours) }\end{array}$ & 51.06 & 49.73 & 0.709 \\
\hline $\begin{array}{l}\text { Mean birth weight } \\
\text { (grams) }\end{array}$ & 3128.00 & 3087.10 & 0.531 \\
\hline $\begin{array}{l}\text { Gender; male gender } \\
\text { (\%) }\end{array}$ & $60(60 \%)$ & $59(59 \%)$ & 0.885 \\
\hline $\begin{array}{l}\text { Reason for test; } \\
\text { bilirubin (\%) }\end{array}$ & $95(95 \%)$ & $97(97 \%)$ & 0.470 \\
\hline State; awake (\%) & $67(67 \%)$ & $69(69 \%)$ & 0.762 \\
\hline
\end{tabular}

NIPS score by both the observers for all neonates were computed. There was a high interobserver reliability (Pearson correlation coefficient, $r=0.977$ ). Both the observers found NIPS score significantly higher in HL group (median NIPS score $=7$ ) than in VP group (median NIPS score $=3.5)$ during the procedure $(p=0.0001)$ [Table 2].

Table 2: The median NIPS scores in HL and VP groups by both the observers

\begin{tabular}{|l|l|l|l|}
\hline & \multicolumn{1}{|c|}{$\begin{array}{c}\text { NIPS score } \\
\text { (median) } \\
\text { (Observer 1) }\end{array}$} & \multicolumn{1}{c|}{$\begin{array}{c}\text { NIPS score } \\
\text { (median) } \\
\text { (Observer 2) }\end{array}$} & p-value \\
\hline $\mathrm{HL}$ & 7 & 7 & \multirow{2}{*}{0.0001} \\
\hline $\mathrm{VP}$ & 3.5 & 3.5 & \\
\hline
\end{tabular}

Regarding physiological response to pain, heart rate (mean \pm SD) before and during procedure were $126.70 \pm 11.82$ and $153.33 \pm 14.15$ respectively in $\mathrm{HL}$ group and $128.92 \pm 14.83$ and $138.32 \pm 17.86$ respectively in VP group. The increase in heart rate in $\mathrm{HL}$ and VP groups were $26.63 \pm 13.31$ and $9.40 \pm 11.27$ respectively. Before the procedure, there was no significant difference in heart rate between both groups $(p=0.243)$ whereas during procedure, there was significant increment in heart rate in both groups $(p=0.0001)$, however the increment was significantly more in $\mathrm{HL}$ group than in VP group ( $p=0.0001$ ). (Fig. 1)

Similarly, oxygen saturations (mean \pm SD) before and during procedure were $95.70 \pm 2.74$ and $89.63 \pm 3.88$ respectively in $\mathrm{HL}$ group and $95.68 \pm 2.46$ and $93.86 \pm 3.13$ respectively in VP group. The decrease in oxygen saturations $\left(\mathrm{SpO}_{2}\right)$ in $\mathrm{HL}$ and VP groups were $6.07 \pm 3.26$ and $1.82 \pm 2.28$ respectively. Before procedure, there was no significant difference in $\mathrm{SpO}_{2}$ between both groups ( $p=0.957$ ) whereas during procedure there was significant decrease in $\mathrm{SpO}_{2}(p=0.0001)$ in both groups, however the fall in $\mathrm{SpO}_{2}$ was significantly more in $\mathrm{HL}$ group than in VP group $(p=0.0001)$ [Fig. 1].

Correlations between NIPS score and physiological parameters of pain (Heart rate and $\mathrm{SpO}_{2}$ ) were calculated by using Spearman rank-order correlations. Significant correlations were found between NIPS and increase in heart rate $(H R)(p=0.0001)$ and NIPS and decrease in $\mathrm{SpO}_{2}(\mathrm{p}=0.0001)$ (table 3$)$.

Table 3: Correlations between NIPS score and physiological parameters of pain

\begin{tabular}{|l|r|r|r|}
\hline Parameter & \multicolumn{1}{|c|}{ NIPS } & \multicolumn{1}{c|}{ HR } & \multicolumn{1}{c|}{ SpO $_{2}$} \\
\hline NIPS & 1.000 & 0.565 & -0.603 \\
\hline $\mathrm{HR}$ & 0.565 & 1.000 & - \\
\hline $\mathrm{SpO}_{2}$ & -0.603 & & 1.000 \\
\hline
\end{tabular}
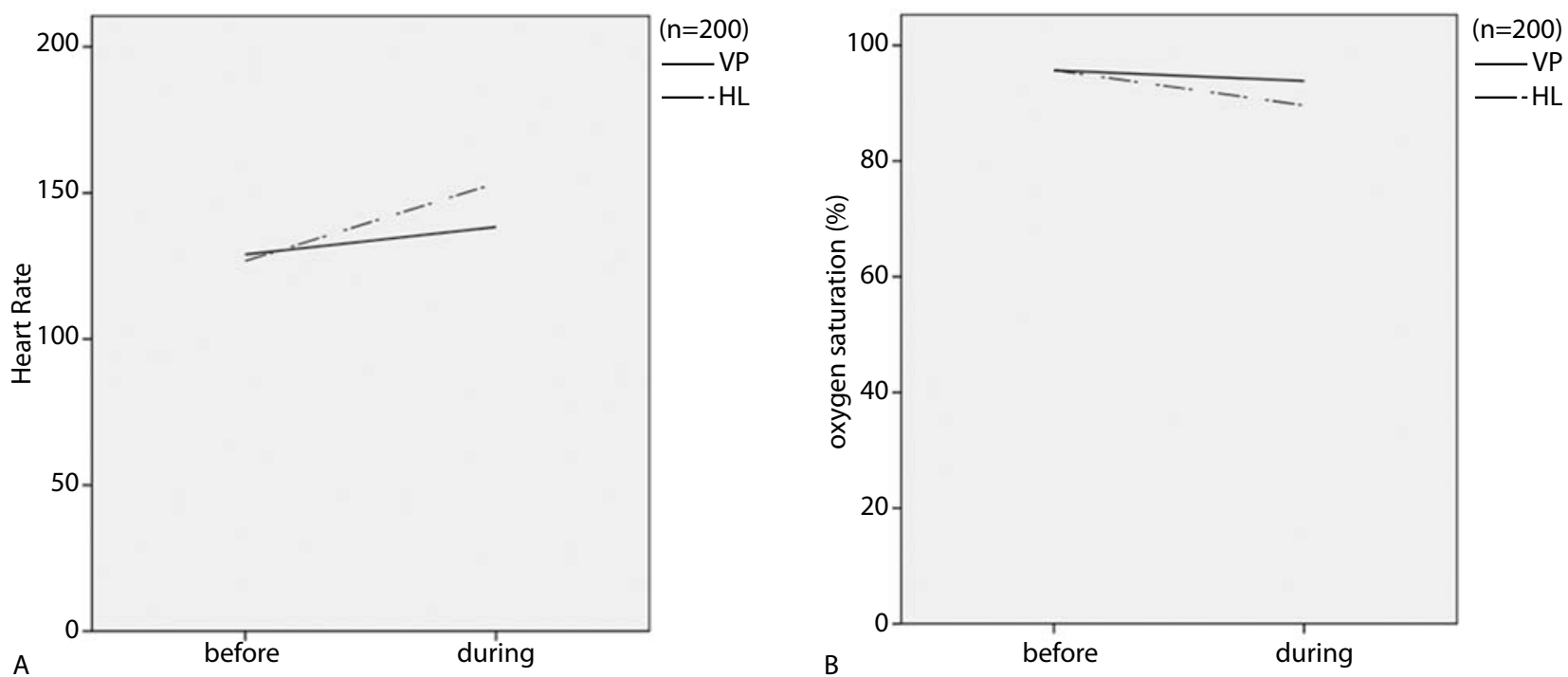

Fig. 1: Comparison of changes in heart rate $(A)$ and $\mathrm{SpO}_{2}(B)$ between $\mathrm{HL}$ and VP groups. 
Table 4: Findings of different studies on pain response to heel lancing and venepuncture

\begin{tabular}{|c|c|c|c|c|c|c|c|c|c|}
\hline \multirow[t]{2}{*}{ Study } & \multicolumn{2}{|c|}{ Sample size } & \multicolumn{2}{|c|}{ Postnatal Age } & \multicolumn{2}{|c|}{$\begin{array}{l}\text { Gender } \\
\text { (\% male) }\end{array}$} & \multicolumn{3}{|c|}{ Pain Score* } \\
\hline & HL & VP & HL & VP & HL & VP & HL & VP & $p$-value ** \\
\hline Shah et $a l^{13}$ & 14 & 13 & $3.1(d)$ & $2.8(d)$ & 57.2 & 61.5 & $\begin{array}{c}5.21^{\#} \\
\text { (NIPS) }\end{array}$ & $\begin{array}{c}2.84 \\
\text { (NIPS) } \\
\end{array}$ & $p<0.001$ \\
\hline \$Larsson et al ${ }^{12}$ & 50 & 50 & $3(d)$ & $4(d)$ & 44 & 55 & $\begin{array}{c}333^{*} \\
\text { (NFCS) }\end{array}$ & $\begin{array}{c}247 \\
\text { (NFCS) }\end{array}$ & $p<0.02(S . L)^{\S}$ \\
\hline \$Ogawa et al' & 25 & 25 & $5(d)$ & $5(d)$ & 48 & 48 & $\begin{array}{c}58^{\epsilon} \\
(\mathrm{NFCS})\end{array}$ & $\begin{array}{c}23 \\
(\mathrm{NFCS})\end{array}$ & $p<0.001$ \\
\hline${ }^{*}$ Eriksson et al20 & 30 & 30 & 4-7 (d) & $4-7(d)$ & 53 & 40 & 8.4 (PIPP) & $\begin{array}{c}6 \\
\text { (PIPP) }\end{array}$ & $p=0.0458$ \\
\hline Present study & 100 & 100 & $51.06(h)^{f}$ & $49.73(h)$ & 60 & 59 & $\begin{array}{c}7 \\
\text { (NIPS) }\end{array}$ & 3.5 (NIPS) & $p=0.0001$ \\
\hline
\end{tabular}

*Pain Score - among various pain rating scales, the compared study used NIPS, NFCS or PIPP

${ }^{* *} p$-value refers to $p$-value of pain scores in different studies

\#Shah et al calculated Mean NIPS score while the present study calculated Median NIPS score

\$both the study Larsson et al and Ogawa et al used NFCS pain rating scale and median NFCS score was calculated

*In Larsson et al study, total range of NFCS scores was $0 \%$ to $600 \%$ as 6 variables of facial action were assessed and the range

for each variable was from $0 \%$ to $100 \%$

$\epsilon$ In Ogawa et al study, all 10 facial actions included in NFCS were assessed each second and the score was accumulated for the following 10 seconds, hence the total score for each assessment point ranged from 0 to 100

${ }^{\S} \mathrm{S} . \mathrm{L}$ - small lancet. In Larsson et al study, neonates receiving heel lance were further assigned into SL and LL (large lancet) groups- 50 in SL and 20 in LL. Only SL group is compared with the present study as the lancet used in SL group which ensured standardized skin depth was almost similar to the present study. The lancet used in LL group was having triangular edge and used hand power to perform heel lancing and without a mechanical device.

${ }^{*}$ Eriksson et al used PIPP as pain rating scale and calculated mean PIPP. This study enrolled term newborns into $4 \mathrm{groups} ; \mathrm{HL}$ and VP with or without glucose, 30 in each group. Here two groups (HL and VP) both without glucose were compared.

${ }^{\mathrm{f}}$ The present study calculated the postnatal age in hours $(\mathrm{h})$ while other study calculated in days (d). Shah et al and the present study calculated mean age while Larsson et al and Ogawa et al calculated median age. Erikkson et al had the neonates of 4-7 days.

\section{Discussion}

Awareness that neonates can perceive pain and it needs to be mitigated did not exist among the health professionals till recently. Pain in neonates had largely been underestimated but the recent studies have conclusively shown that neonates exhibit pain response which should be mitigated by any means $\mathbf{s}^{7,15,16}$.

The present study has clearly demonstrated neonates perceive pain and the intensity of pain varies with magnitude of invasion. NIPS and physiological changes ( $\mathrm{HR}$ and $\mathrm{SpO}_{2}$ ) during procedure successfully showed neonates perceive pain in both the procedures; heel lancing and venepuncture $(p=0.0001)$, but the intensity of pain was significantly more in neonates of $\mathrm{HL}$ than those of VP $(p=0.0001)$.

Table 4 summarizes the findings of different studies on pain response to both methods of collecting blood samples in neonates. The present study with the largest sample size has shown findings consistent with similar studies in the past.
All the studies above showed that pain response was significantly higher $(p<0.05)$ in $\mathrm{HL}$ than in VP. Different studies have used different but valid pain assessment tools; all are consistent with the result.

In the present study, in response to both heel lance and venepuncture, neonates showed significant changes in heart rate and oxygen saturation as compared to their respective pre-procedure parameters $(p=0.0001)$; however magnitude of changes in both heart rate and oxygen saturation were significantly more in $\mathrm{HL}$ group than in VP group $(p=0.0001)$.

In response to venepuncture, Amar M. Taksande, ${ }^{14}$ Singh et $\mathrm{al}^{16}$ and Bauer et $\mathrm{al}^{21}$ reported a significant increase in heart rate and decrease in oxygen saturation which are consistent with the present study. In response to heel lancing, Weissman et $\mathrm{al}^{7}$ and Lindh et $\mathrm{al}^{22}$ reported a significant increase in heart rate. Hyesang et $\mathrm{al}^{23}$ reported a significant increase in heart rate and decrease in oxygen saturation to heel lancing. All of these findings are consistent with the findings of the present study. 
Pereira et $\mathrm{al}^{24}$ reported a significant change in physiological response even to a painless but distressing procedure like alcohol swabbing; however, the magnitude of change was less in such procedure than in a painful (tissue breaking) one. Porter et $\mathrm{al}^{25}$ reported the significant changes in physiological and behavioral measures in response to painful procedures and the magnitude of response generally increased with increased procedural invasiveness. Thus the changes in physiological response vary with degree of invasiveness and more the change in physiological response, more the neonate is having pain. The present study showing significantly more change in physiological response to heel lancing than venepuncture, reflects the former being more painful than the latter. Furthermore, the significant correlations between NIPS and the physiological parameters (changes in $\mathrm{HR}$ and $\mathrm{SpO}_{2}$ ) support that heel lancing is more painful than venepuncture.

However, the present study has certain limitations: it does not compare the change in physiological parameters ( $\mathrm{HR}$ and $\mathrm{SpO}_{2}$ ) during the post-procedure recovery state. A record of that state would have provided better information about the duration of pain and its impact on physiological parameters. The study does not account for number of pricks required, duration to collect required volume of blood and any manipulation like squeezing for drawing blood, hence the study could not comment regarding efficacy of the procedure. Further studies are recommended regarding the comparative efficacy of each procedure.

The study stresses that prevention of pain or its management should not be neglected whatever may be the age. Thus, it is recommended to use less painful method in every nursery/NICU for routine procedures, i.e., for blood sampling. Use of venepuncture is recommended instead of heel lancing. Further studies are recommended regarding the measures for pain mitigation like feeding a newborn, giving $30 \%$ sucrose solution etc. prior to procedure. Last but not the least, wider dissemination of information regarding the perception of pain in neonates and a necessity to take steps to minimize such pain should be a part of education of health professionals including pediatricians who care for neonates.

To the best of our knowledge, this is the first study of its kind to be carried out in our centre and in Nepal as well.

\section{Conclusion}

This study demonstrates that neonates perceive pain which can objectively be detected by behavioral rating scale 'NIPS' and changes in such physiological parameters as heart rate and oxygen saturation. The study concludes that venepuncture is less painful than heel lancing for blood sampling in neonates.

Acknowledgements: I am indebted to Mr. Megha Raj Banjara, Department of Microbiology for his valuable help during statistical analysis. My sincere thanks to all the faculties of department of Paediatrics, resident doctors, nursing staffs of maternity and neonatal unit of Institute of Medicine.

\section{Funding: Nil \\ Conflict of interests: None}

Permission from IRB: The study is permitted and validated by departmental research board from Department of Paediatrics, IOM, TUTH.

\section{References}

1. William T. Zempsky, Neil L. Schechter. What's New in the Management of Pain in Children. Pediatr in Review 2003;24:337-48.

2. K. J. S. Anand and the International Evidence-Based Group for Neonatal Pain. Consensus Statement for the Prevention and Management of Pain in the Newborn. Arch PediatrAdolesc Med 2001;155:173-80.

3. P J Mathew and J L Mathew. Assessment and management of pain in infants. Postgrad Med $J$ 2003;79:438-43.

4. Alberto Loizzo, Stefano Loizzo and Anna Capasso. Neurobiology of Pain in Children: An Overview. The Open Biochem J;2009;3:18-25.

5. Prevention and management of pain in the neonate. An update. Adv Neonatal Care 2007; 7(3):151-60.

6. Heather D. Hadjistavropoulos, Kenneth D. Craig, Ruth Eckstein Grunau, Michael F. Whitfield. Judging pain in infants: behavioural, contextual, and developmental determinants. Pain 1997;73(3):31924.

7. Weissman A, Aranovitch M, Blazer S, Zimmer EZ. Heel-Lancing in Newborns: Behavioral and Spectral Analysis Assessment of Pain Control Methods. Pediatrics 2009;124;e921-e926.

8. Sweet SD, McGrath PJ. Physiological measures of pain. In: Finley GA, McGrath PJ, editors. Measurements of pain in infants and children. Seattle: IASP Press 1998:59-82.

9. Grunau RVE, Johnston CC, Craig KD. Neonatal facial responses to invasive and non-invasive procedures. Pain 1990;42:295-305. 
10. Hadjistavropoulos HD, Craig KD, Grunau RVE, Johnston CC. Judging pain in newborns: facial and cry determinants. J Pediatr Psychol 1994;19:485-91.

11. Shah VS, Ohlsson A. Venepuncture versus heel lance for blood sampling in term neonates. Cochrane Database of Systematic Reviews2007, Issue 4. Art. No.: CD001452.

12. Björn A. Larsson, GunnillaTannfeldt, Hugo Lagercrantz and Gunnar L. Olsson. Venipuncture Is More Effective and Less Painful Than Heel Lancing for Blood Tests in Neonates. Pediatrics 1998;101;882886.

13. Vibhuti S Shah, Anna Taddio, Sian Bennett, et al. Neonatal pain response to heel stickvsvenepuncture for routine blood sampling. Arch Dis Child Fetal Neonatal Ed 1997;77: F143-F144.

14. Amar M. Taksande, K.Y. Vilhekar, M. Jain and D. Chitre. Pain Response of Neonates to Venipuncture. Indian J Pediatr 2005;72(9):751-53.

15. Fran Lang Porter, Cynthia M. Wolf and J. Philip Miller. Procedural Pain in Newborn Infants: The Influence of Intensity and Development. Pediatrics 1999;104;e13.

16. Harmesh Singh, Daljit Singh, R.K. Soni. Comparison of pain response to venepuncture between term and preterm neonates. Indian Pediatr 2000;179-181.

17. DP. Barker and $\mathrm{N}$ Rutter. Exposure to invasive procedures in neonatal intensive care unit admissions. Arch Dis Child Fetal Neonatal Ed. 1995 Jan;72(1):F47-F48.

18. Lawrence J, Alcock D, McGrath P, Kay J, McMurray SB, Dulberg C. The development of a tool to assess neonatal pain. Neonatal Network 1993;12:59-66.
19. S Ogawa, T Ogihara, E Fujiwara, K Ito, M Nakano, S Nakayama, T Hachiya, N Fujimoto, H Abe, S Ban, E Ikeda, $\mathrm{H}$ Tamai. Venepuncture is preferable to heel lance for blood sampling in term neonates. Arch Dis Child-Fetal and Neonatal Ed 2005;90:F432-F436.

20. Mats Eriksson, Maria Gradin, Jens Schollin. Oral glucose and venepuncture reduce blood sampling pain in newborns. Early Human Dev 1999;55:211218.

21. Bauer, Karl; Ketteler, Jörg; Hellwig, Magdalena; Laurenz, Maren; Versmold, Hans. Oral Glucose before Venepuncture Relieves Neonates of Pain, but Stress Is Still Evidenced by Increase in Oxygen Consumption, Energy Expenditure, and Heart Rate. Pediatr Research 20041;55(4):695-700.

22. Viveca Lindh, Urban Wiklund, StellanHåkansson. Heel lancing in term new-born infants: an evaluation of pain by frequency domain analysis of heart rate variability. Pain 1999;80:143-148.

23. Hyesanglm, Eunjung Kim, Eunsook Park, Kyungsuk Sung and Wonoak Oh. Pain Reduction of Heel Stick in Neonates: Yakson Compared to Non-nutritive Sucking. J Trop Pediatr 2008;54 (1):31-35.

24. Andrea Lübe de S Thiago Pereira, Ruth Guinsburg, Maria Fernanda Branco de Almeida, Ana Cristina Monteiro, AméliaMiyashiroNunes dos Santos, Benjamin Israel Kopelman. Validity of behavioral and physiologic parameters for acute pain assessment of term newborn infants. Sao Paulo Med J/Rev Paul Med 1999;117(2):72-80.

25. Fran Lang Porter, Cynthia M. Wolf and J. Philip Miller. Procedural Pain in Newborn Infants: The Influence of Intensity and Development. Pediatrics1999;104:e13.

\section{How to cite this article?}

Shrestha M, Adhikari RK. Comparison of Pain Response to Venepuncture Versus Heel Lance Blood Sampling in Term Neonates. J Nepal Paediatr Soc 2012;32(2):99-104. 